\title{
Record Charge Carrier Diffusion Length in Colloidal Quantum Dot Solids via Mutual Dot-To-Dot Surface Passivation
}

\author{
Graham H. Carey, Larissa Levina, Riccardo Comin, \\ Oleksandr Voznyy, Edward H. Sargent
}

Version Post-Print/Accepted Manuscript

Citation Carey, G., Levina, L., Comin, R., Voznyy, O., \& Sargent, E. (2015).

(published version) Record Charge Carrier Diffusion Length in Colloidal Quantum Dot

Solids via Mutual Dot-To-Dot Surface Passivation. Advanced Materials, 27(21), 3325-3330. http://dx.doi.org/10.1002/adma.201405782

\begin{abstract}
Publisher's Statement This is the peer reviewed version of the following article: Carey, G., Levina, L., Comin, R., Voznyy, O., \& Sargent, E. (2015). Record Charge Carrier Diffusion Length in Colloidal Quantum Dot Solids via Mutual Dot-To-Dot Surface Passivation. Advanced Materials, 27(21), 33253330, which has been published in final form at http://dx.doi.org/10.1002/adma.201405782. This article may be used for non-commercial purposes in accordance with Wiley Terms and Conditions for Self-Archiving.
\end{abstract}

How to cite TSpace items

Always cite the published version, so the author(s) will receive recognition through services that track citation counts, e.g. Scopus. If you need to cite the page number of the TSpace version (original manuscript or accepted manuscript) because you cannot access the published version, then cite the TSpace version in addition to the published version using the permanent URI (handle) found on the record page. 
DOI: $10.1002 /(($ please add manuscript number $))$

Article type: Communication

\section{Record Charge Carrier Diffusion Length in Colloidal Quantum Dot Solids via Mutual Dot-to-Dot Surface Passivation}

Graham H. Carey, Larissa Levina, Riccardo Comin, Oleksandr Voznyy, Edward H. Sargent*

G. H. Carey, Dr. L. Levina, Dr. R. Comin, Dr. O. Voznyy, Prof. E. H. Sargent

Department of Electrical and Computer Engineering

University of Toronto

10 King's College

Toronto, Ontario, M5S 3G4, Canada

E-mail: ted.sargent@utoronto.ca

Keywords: colloidal quantum dots, surface passivation, diffusion length, surface-to-volume ratio

Colloidal quantum dots (CQDs) offer a powerful platform for the fabrication of optoelectronic devices including photodetectors ${ }^{[1]}$, photovoltaics ${ }^{[2-4]}$, and light-emitting diodes ${ }^{[5,6]}$. A key advantage of CQDs resides in the quantum confinement effect wherein the bandgap and optoelectronic properties can be tailored simply by adjusting the size of nanoparticle used ${ }^{[7-9]}$.

This flexibility brings with it a challenge compared to the case of typical bulk polycrystalline semiconductors. The CQD diameter (in, for example, $\mathrm{PbS}$ and $\mathrm{PbSe}$ ) to achieve $\mathrm{a} \sim 1.0-1.3 \mathrm{eV}$ bandgap is in the range of $3-4 \mathrm{~nm}^{[10,11]}$. As a result, a large proportion of atoms making up the quantum dot are surface atoms that require careful termination in light of the crucial influence of nanocrystal surfaces on passivation and confinement ${ }^{[12,13]}$. Much of the work in this field has focused on passivating maximally these surface sites using ligands. Indeed, innovative strategies including both molecular ${ }^{[3,14-17]}$ and atomic ${ }^{[4,18,19]}$ ligands have recently led to considerable advances in in-gap trap state density and consequently solar cell performance. While considerable progress has been made, quantum dot surface passivation remains a significant limiter of film optoelectronic properties and device performance ${ }^{[20]}$.

Recent reports of partially-fused quantum dot systems ${ }^{[21,22]}$ hint at a possible new passivation strategy. Rather than passivate every surface site using extraneous chemical 
additives, we would seek to combine chemical passivation with partial fusing of certain surfaces presented by adjacent quantum dots. Our strategy (depicted in Figure 1a) would unite dot-to-dot surface-coupled self-passivation (to reduce the overall surface-to-volume ratio within the quantum dot solid) with strong chemical passivation of the remaining surface sites. We show herein that reducing the available surface area while strongly passivating remaining sites led to a very low density of electronic traps. We further show that, at the same time as excellent electronic transport was achieved, impressively long-lived excited-state lifetimes were simultaneously observed. The combination of excellent transport and reduced trapping led to the longest reported diffusion length $\underline{s}$ observed in a colloidal quantum dot solid.

a
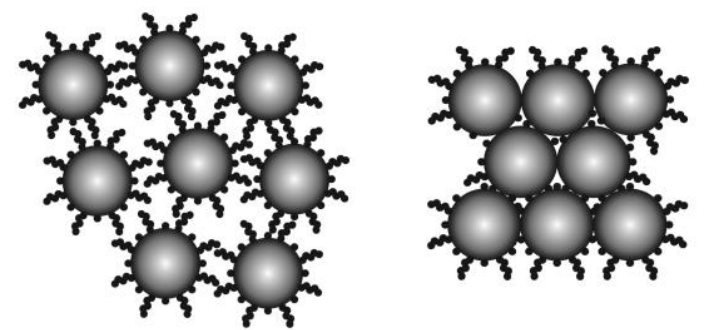

b

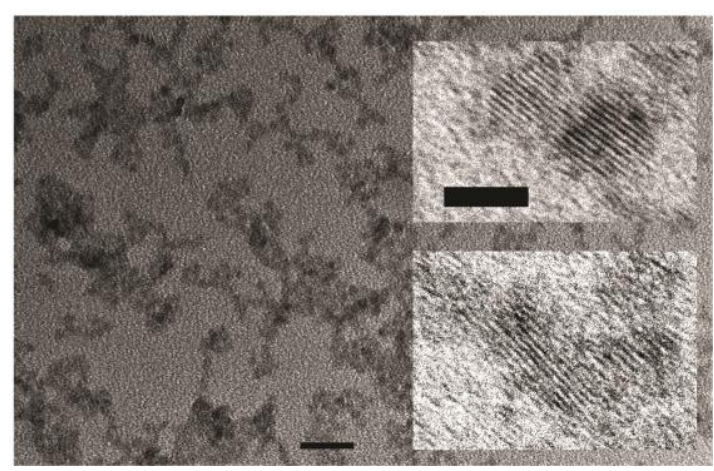

Figure 1. a) Schematic of a typical collection of colloidal quantum dots after ligand exchange when initially capped with oleic acid and chloride (left) or a short primary amine and chloride (right). Amine-capped dots allow partial fusing, reducing total available surface sites in a film. b) Transmission electron microscope image showing partial quantum dot fusing occurring - insets show prominent instances of two or more dots fusing. Scale bars indicate $20 \mathrm{~nm}$ in the overview and 5. $\mathrm{nm}$ in the two inset images.

The aforementioned recent reports, in which quantum dots were partially connected, did not incorporate, in combination with their partial connection strategies, the most effective 
passivation strategies. Indeed, this was not an option, for the surface fusing was based on harsh processing conditions that strippeding off all passivating ligands.

We therefore devised instead a new process that could allow the combination of partial facet fusing and-to be combined with excellent chemical surface passivation. It is based on a combination of a strongly bound atomic ligand, which serves to provideing efficient passivation, and a more labile organic ligand, used only to ensure steric colloidal stabilization. Amine treatments have recently been deployed to create well-packed, connected, and highly conductive arrays of quantum dots ${ }^{[22]}$. We therefore developed a synthesis method designed to produce $\mathrm{PbS}$ quantum dots capped with amine ligands instead of oleic acid. The synthesis design was informed by recent reports of highly passivated, amine-stabilized CdSe quantum $\operatorname{dots}^{[23-25]}$. The synthesis is described in full in the experimental section; as shown in Scheme 1, CQDs are initially synthesized with oleic acid capping ligands, which are immediately replaced with chloride atomic ligands and tributylphosphine $\left(\mathrm{PBu}_{3}\right)$. A mixture of $\mathrm{n}$ hexylamine and n-octylamine is then used to replace the $\mathrm{PBu}_{3}$, balancing the need for ligands long enough to stabilize the nanocrystals in a standard organic solution, yet short enough to enable partial fusing during film formation. The ligand exchange was tracked via Fourier transform infrared spectroscopy (FTIR); Figure S1, Supporting Information, cempares $\underline{\text { characterizes one representative a-dot batch at each stage of the exchange. In contrast with the }}$ previously reported exchange using $\mathrm{CdSe} C \mathrm{CQDs}$, oleate is evident at all stages of the $\mathrm{PbS}$ CQD exchange until the final amine replacement step. X-ray photoelectron spectroscopy (XPS) measurements were performed on pristine, dropcast films of the amine-exchanged $\mathrm{PbS}$ CQDs (Figure S2, Supporting Information), showing the presence of chloride ligands (Figure $\underline{\mathrm{S} 2 \mathrm{a} \text { ), and the full elimination of } \mathrm{PBu}_{3} \text { and oleate (Figure } \mathrm{S} 2 \mathrm{~b} \text { and c). We hypothesize that the }}$ $\underline{\mathrm{PBu}}_{3}$ and $\mathrm{Me}_{3} \underline{\mathrm{Si}-\mathrm{Cl} \text { exchange steps partially replace oleic acid on the surface, providing initial }}$ surface chloride ligands and facilitating the final, full amine exchange required for subsequent 
facet fusing. before and after ligand exchange, showing full ligand exchange to amine

eapping.

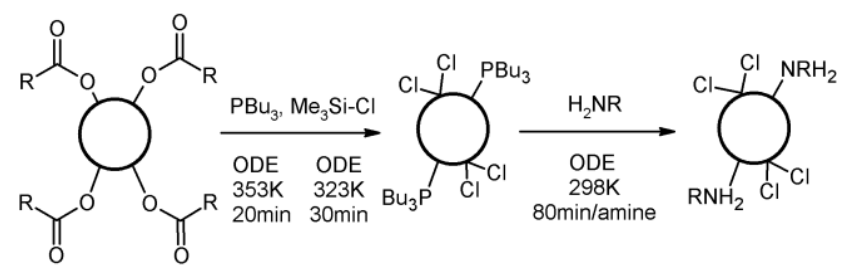

Scheme 1. Synthesis of $\mathrm{PbS}-\mathrm{RNH}_{2}-\mathrm{Cl}$ colloidal quantum dots (mutually-passivated $\mathrm{PbS}$ ).

Using HRTEM, we investigated whether any quantum dot fusing occurred in the resultant films following film deposition and ligand exchange using 3-mercaptopropionic acid (MPA). Figure 1b shows HRTEM images that, even at the very low concentrations required to generate a sub-monolayer of quantum dots, evidence partially fused dot clusters. The insets in Figure 1b show specific instances of two or more dots that have fused, and alignment in diffraction fringes is evident among multiple adjacent quantum dots. Such alignment (oriented attachment) is a prerequisite for fusion to produce mutual passivation.

Further evidence of partial surface fusing was observed in full ligand-exchanged films. Films were fabricated using a layer-by-layer spin-coating approach; each layer of aminecapped $\mathrm{PbS}$ quantum dots was place-exchanged by treating tsing-with a solution of MPA mixed with a low concentration $(0.2 \mathrm{mM})$ of $\mathrm{CdCl}_{2}$. The goal of the inorganic metal halide salt was to retain and indeed improve halide passivation. Comparing absorption in solution to film absorption after ligand exchange (Figure 2a), we note two evident features-are evident. First, significant broadening has occurred, with a larger long-wavelength contribution after exchange, consistent with fusing within the film. Second, despite evidence of fusing, the excitonic feature is still relatively strong, indicating that the exchange process has not eliminated the quantum confinement effect from the resulting film.

X ray photoelectron spectroscopy (XPS $)$ measurements provide further support for fusing, as well as evidence of improved overall passivation. Control quantum dots (oleic 
$\underline{\text { acid/CdCl}} 2$ passivated $\mathrm{PbS}$ ) were compared with mutually passivated quantum dots

(amine/chloride passivated $\mathrm{PbS}$ ). Each film was treated with MPA and $\mathrm{CdCl}_{2}$ as described $\underline{\text { above, to maintain an equivalent number of chloride passivation steps; this is not expected to }}$ $\underline{\text { significantly impact the surface chemistry of control dots compared with a typical, MPA-only }}$ exchange. Figure S2d, Supporting Information, provides evidence from XPS measurements that the addition of $\mathrm{CdCl}_{2}$ to the ligand exchange step does not impact the amount of chloride in a control dot film. Comparing mutually-passivated to control CQD films, it is observed that Tthe thiol contribution to the sulfur $2 p$ signal (left-hand contribution to sulfur signal in Figure 2b) is significantly reduced ${ }_{2}-$ compared to a control film of $\mathrm{MPA} / \mathrm{CdCl}_{z}$-exchanged, initially oleic acid and $\mathrm{CdCl}_{2}$ passivated $\mathrm{PbS}$ quantum dots (the same dots used for the highest performance MPA capped films to date, hereafter referred to as "control dots" for simplicity). Reduced presence of thiol is consistent with reduced available surface area $s$, $\underline{B}$ y contrast, the chloride $2 p$ signal is unchanged between the two treatment types, since whereas atomic (chloride) ligands are less impacted by the steric limitation of fused dot surfaces $;$ the chloride 2p signal is unchanged between the two treatment types.

a

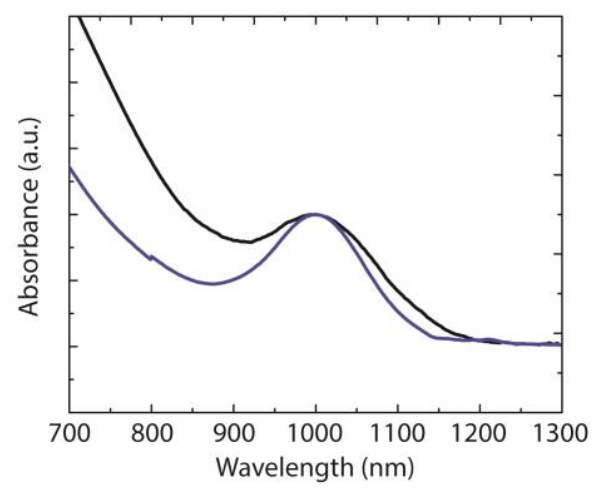

b

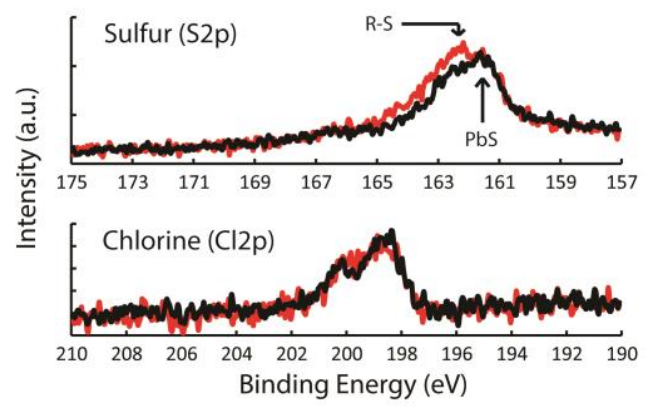


Figure 2. a) Absorbance (normalized to exciton peak) of amine-capped PbS quantum dots in solution (blue) and in film after ligand exchange with 3-mercaptopropionic acid and $\mathrm{CdCl}_{2}$ (black). b) X-ray photoelectron spectra examining sulfur and chlorine characteristics in films of oleic acid (red) or amine (black) capped quantum dots exchanged with 3-mercaptopropionic acid and $\mathrm{CdCl}_{2}$. Arrows in the sulfur spectrum indicate the positions of lead-bound sulfur and ligand sulfur.

We interpret these stoichiometry data as promising with respect to the prospects for improved passivation. Fewer surface sites are available in the fused-surface case, since these surfaces are already occupied. Thus, having an equivalent density of chloride atoms in the film suggests a larger number of passivating halide anions per surface atom of the quantum dots, i.e. it points to the possibility of increased passivation density of the exposed facets.

We now turn to studies of minority carrier diffusion length $\left(\mathrm{L}_{D}\right)$, a key parameter most urgently in need of major progress in the field of CQD photovoltaics. We have shown recently that extending diffusion length will rely principally on reducing the density of deep electronic traps in CQD solids, while improving dot-to-dot transport will also offer advantage in the best present-day films ${ }^{[20]}$. We employed transient photoluminescence (PL) measurements to determine carrier diffusion lengths, for these methods have been extensively practiced in organic ${ }^{[26]}$ and perovskite ${ }^{[27,28]}$ semiconductors in very compelling recent reports. We would thereby directly compare control vs. fused-and-passivated CQD films, and also benchmark our results to analogous reports in other materials systems.

The PL-based derivation of diffusion length relies on evaluating variation in the PL lifetime for pristine films (of known thickness) with and without a photoexcited-statequenching interface. The dynamics and times scales of photogenerated carriers are subsequently interpreted within a 1D diffusion model in order to estimate the carrier lifetime and diffusion length.

CQD solids were formed via spin-coating on glass and were ligand-exchanged as described abovewith MPA (control) or a mixture of MPA and $\mathrm{CdCl}_{2}$ (mutually-passivated), as described above. Unquenched films were prepared on bare glass, with film thicknesses of 
roughly $250 \mathrm{~nm}$ to ensure adequate PL signal and accurate carrier lifetime measurements $\div$ As shown in Figure S3, Supporting Information, single layer films exhibited an abnormally fast decay time, potentially attributable to cracks and voids-which are infilled by subsequent layers, whereas thicker films exhibited minimal thickness dependence on decay time due to the infilling of cracks by subsequently deposited layers- thinner unquenched films exhibited some thickness dependence, potentially as a result of variable quenching at the glass/quantum dot interface. Quenched films were prepared by spin-coating an electron-accepting layer of phenyl- $\mathrm{C}_{61}$-butyric acid methyl ester (PCBM) on bare glasstitanium dioxide, then depositing a thin-two quantum dot layers $(\underline{502} 25+/-5 \mathrm{~nm}$ and $\underline{735}+/-5 \mathrm{~nm}$ for the control and mutuallypassivated films, respectively). The thickness was chosen to fall below expected diffusion length in each case in order to ensure a sizeable quenching effect. In all cases, the pulsed excitation source $(633 \mathrm{~nm})$ was incident on the CQD film side. Steady-state photoluminescence measurements (Figure $S \underline{4} \underline{z}$, Supporting Information) were used to determine the emission wavelength of interest for subsequent decay measurements $(1110 \mathrm{~nm}$ for control dots, $1225 \mathrm{~nm}$ for mutually-passivated dots).

Time-resolved PL decay measurements are shown in Figure 3, along with twocomponent exponential fits to each data set, representing a fast exciton dissociation time constant and a slower transport-assisted recombination lifetime. The fast, but not resolutionlimited, exponential component was accurately determined by fitting data measured on a smaller time window (Figure S $\underline{5}\}$, Supporting Information). The carrier lifetimes for each case are reported and compared in Table 1; even before evaluating the results within the framework of the diffusion model, one readily observes that the mutual-passivation method leads to a doubling of excited statecarrier lifetime compared to the control. At the same time it produces a significantly shorter quenching lifetime, suggesting the sought-after improved transport. 

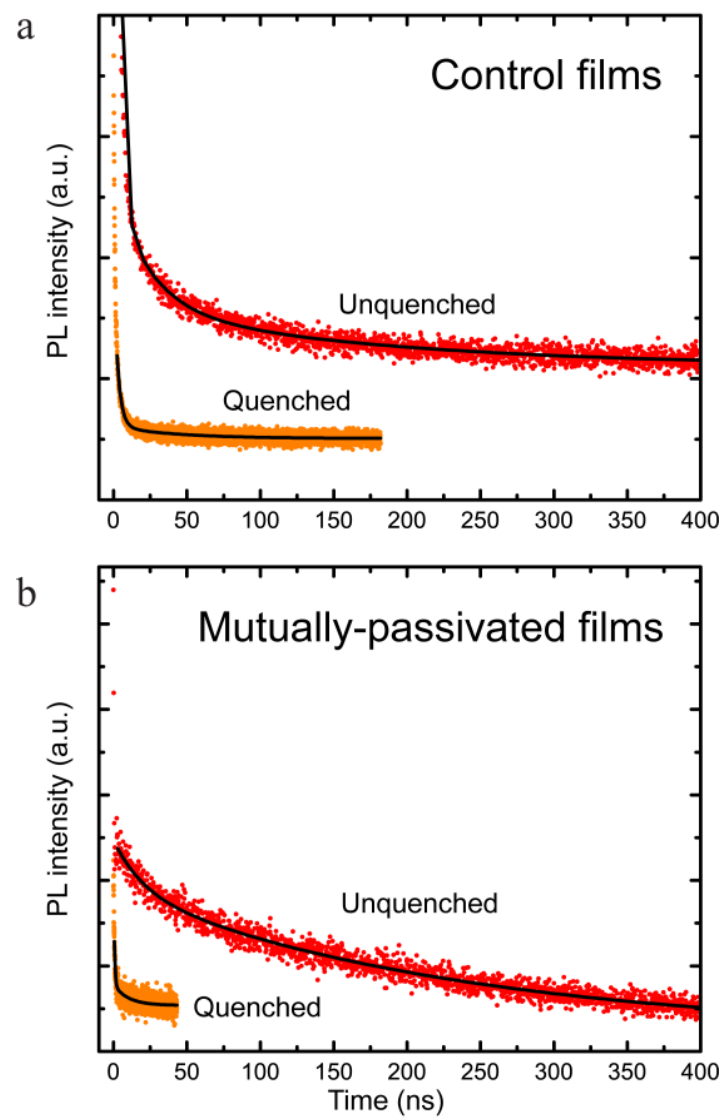

Figure 3. Photoluminescence lifetime measurements of MPA-exchanged films of a) control $\mathrm{PbS}$ quantum dots and b) mutually-passivated $\mathrm{PbS}$ quantum dots. Red points represent data for thick (>200nm) films on glass, while orange points represent thin films (2550 and $3575 \mathrm{~nm}$, respectively) on a quenching layer of PCBM/titanium dioxide.

Table 1. Electrical carrier transport figures of merit.

\begin{tabular}{lccccc}
\hline & $\begin{array}{c}\text { Unquenched } \\
\text { Lifetime [ns] }\end{array}$ & $\begin{array}{c}\text { Quenched } \\
\text { Lifetime } \\
{[\mathrm{ns}]}\end{array}$ & $\begin{array}{c}\text { Diffusion } \\
\text { Length } \\
{[\mathrm{nm}]}\end{array}$ & $\begin{array}{c}\text { Mobility } \\
{\left[\mathrm{cm}^{2} \mathrm{~V}^{-1} \mathrm{~s}^{-1}\right]}\end{array}$ & $\begin{array}{c}\text { Exciton } \\
\text { Dissociation } \\
\text { Rate }\left[\mathrm{ns}^{-1}\right]\end{array}$ \\
\hline Control & $148+/-4$ & $\begin{array}{c}2 \underline{4} 0.9+/- \\
\underline{3} 4.5\end{array}$ & $\underline{\underline{7038+/-}}$ & $1 \times 10^{-2}$ & $2.0+/-0.1$ \\
$\begin{array}{l}\text { Mutually- } \\
\text { passivated }\end{array}$ & $268+/-5$ & $\underline{117.6+/-}$ & $\underline{\underline{230} 145+/-}$ & $8 \times 10^{-2}$ & $3.7+/-0.1$ \\
\hline
\end{tabular}

Full details on the diffusion model applied to the PL decay results can be found in previous reports ${ }^{[28]}$; in brief, the dynamics are modeled using a one-dimensional diffusion equation (Equation 1):

$\frac{\partial n(x, t)}{\partial t}=D \frac{\partial^{2} n(x, t)}{\partial x^{2}}-k(t) n(x, t)$

where $\mathrm{D}$ is the diffusion coefficient, and $\mathrm{k}$ represents the unquenched PL decay rate. Equation 1 is solved for the time- and depth-dependent carrier concentration by assuming appropriate 
boundary conditions, i.e. that all carriers at the quantum dot/PCBM interface are quenched, leading to the condition $\mathrm{n}(\mathrm{L}, \mathrm{t})=0$, where $\mathrm{L}$ is the film thickness, and that the carrier population outside the film is zero, i.e. $n(x, t)=0$, for $x<0$. Additionally, the initial excitation distribution can be written as $n(x, 0)=n_{0} \exp (-\alpha x)$, where $\alpha$ is the absorption coefficient at the excitation wavelength. Absorption coefficients for each film were extracted from Figure Sㅁ4, Supporting Information.

As previously reported, this model can be simplified to a plot of diffusion length in units of film thickness against the ratio between quenched and unquenched PL lifetimes (Figure S75, Supporting Information). Plotting the two observed lifetime ratios on this curve leads to an estimated diffusion length of $\underline{70} 38+/-\underline{109} \mathrm{~nm}$ for the control, and of $\underline{230145+/-}$

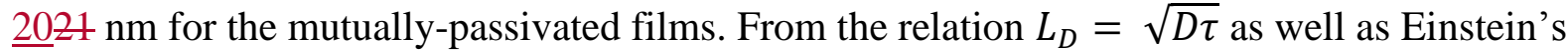
relation (Equation 2), the effective electron mobility can also be estimated for each film. Diffusion length and mobility are reported together with carrier lifetime measurements and estimated exciton dissociation rates in Table 1.

$$
D=\frac{\mu k_{B} T}{q}
$$

The threenearly four-fold improvement in diffusion length compared to control films represents the highest reported diffusion length to date in colloidal quantum dot solids. This measured value is comparable to diffusion lengths in those perovskite films typically utilized in mesoporous electrodes, though shorter than the longest $L_{D}$ values reported and employed in planar perovskite architectures.

A compelling example application of a significantly longer diffusion length is the realization of a photovoltaic device that can exploit a notably thicker light-absorbing layer, enabling a higher current density and maintaining fill factor. For control films, it has been previously reported that the optimal film thickness is typically in the range of $300 \mathrm{~nm}$, beyond which any minimal gains in current density are more than offset by a loss in fill factor. By 
contrast, devices prepared using the mutual-passivation method were found to have an optimal thickness of $600 \mathrm{~nm}$. Electrical characterization of a hero device is shown in Figure 4a; fill factor for the thick device is comparable to that of standard-thickness control devices ( $58 \%$ fill factor), while the additional $300 \mathrm{~nm}$ of absorptive material allows the generation of just below $30 \mathrm{~mA} / \mathrm{cm}^{2}$ in current density (confirmed by external quantum efficiency measurements, Figure 4b). While open-circuit voltage is somewhat lower than control devices (likely explained by the red-shift due to fusing observed in Figure 4b), the power conversion efficiency is $9.2 \%$.
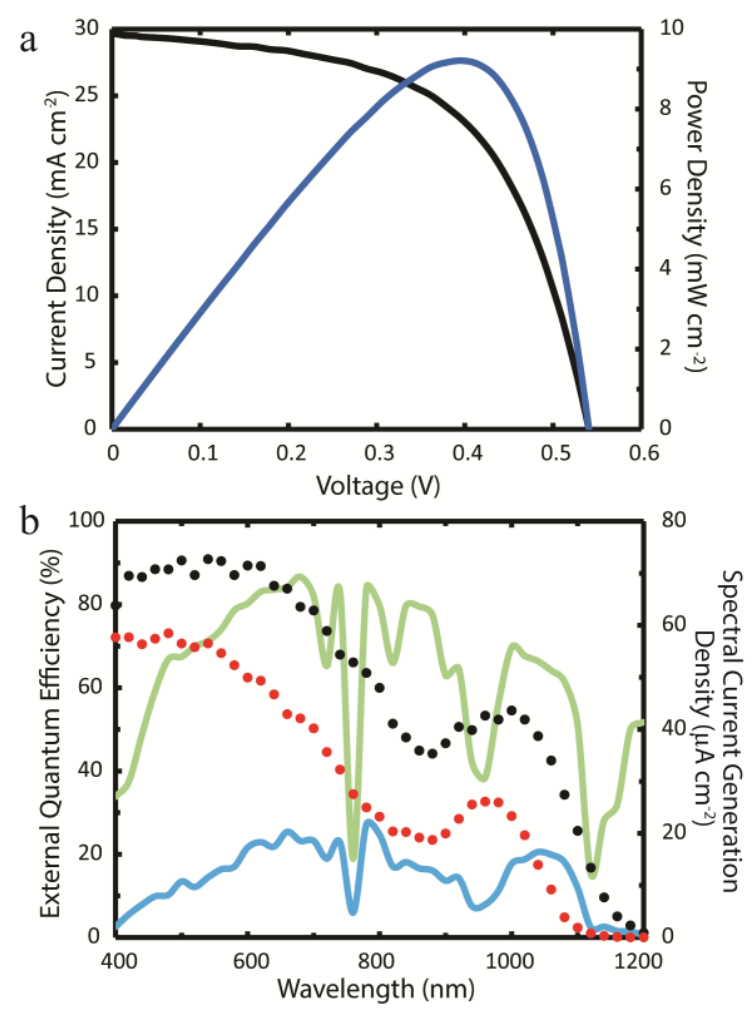

Figure 4. a) Current (black) and power (blue) density versus applied bias measurements for a high diffusion length film (thickness $600 \mathrm{~nm}$ ), with maximum power point power conversion efficiency of $9.24 \%$. b) External quantum efficiency measurements for thickness-optimized control films (red spots, calculated current $21.6 \mathrm{~mA} \mathrm{~cm}^{-2}$ ) and mutually-passivated films (black spots, calculated current $29.5 \mathrm{~mA} \mathrm{~cm}^{-2}$ ). Total spectral current generation density for a hypothetical 100\% EQE device is shown in light green; additional spectral current density gained by the mutually-passivated devices compared to control is shown in light blue.

The dot-to-dot mutual surface passivation approach, when combined with the best available methods in quantum dot chemical passivation, leads to the formation of films with 
diffusion lengths fully three times longer than previously-reported quantum dot solids. Applying this materials processing approach to photovoltaic devices allows for thicker optimized films, with significant increases in photocurrent and negligible fill factor loss. Additionally, the approach offers a robust platform for interesting future applications; low trap density and long diffusion length are desirable qualities when constructing highperformance current-matched tandem photovoltaic devices, either fully CQD-based or as efficient IR conversion in conjunction with crystalline semiconductor cells.

\section{Experimental Section}

Colloidal Quantum Dot (CQD) Synthesis: Control PbS quantum dots were synthesized according to a previously published method ${ }^{[29]}$, using a previously reported in-synthesis halide treatment $^{[3]}$. PbS CQD for mutual passivation studies were synthesized using a slight variant of the aforementioned method: after the growth of oleic acid-capped CQD of desired size (omitting the in-synthesis $\mathrm{CdCl}_{2}$ treatment), tributylphosphine was injected during the reaction cooling process, with injection at $80^{\circ} \mathrm{C}$ and 20 minute reaction time.

Trimethylchlorosilane was injected at $50^{\circ} \mathrm{C}$, with a 30 minute reaction time. After the reaction vessel cooled to $30^{\circ} \mathrm{C}$, the quantum dot solution aggregated; the supernatant was removed and the reaction vessel was refluxed with argon. Subsequently, n-hexylamine was injected (stirring 80 minutes) followed by n-octylamine for an additional 80 minutes stirring. Following full reaction, the CQD solution was precipitated with methyl acetate, dried for 16 hours under mild vacuum, and redispersed at roughly $100 \mathrm{mg} \mathrm{mL}^{-1}$ in octane. All reagents were purchased from Sigma Aldrich.

CQD Film Fabrication and Ligand Exchange: CQD films were prepared on glass (for spectroscopy and PL studies) or $\mathrm{TiO}_{2}$ electrodes (for photovoltaic devices; $50 \mathrm{~nm}$ of $\mathrm{TiO}_{2}$ sputtered on fluorine-doped tin oxide (FTO)-coated glass) using layer-by-layer spin-coating deposition under ambient conditions. Control films were deposited from a $50 \mathrm{mg} \mathrm{mL}^{-1}$ 
solution of quantum dots in octane at $2500 \mathrm{rpm}$ to obtain layers of roughly $25 \mathrm{~nm}$ thickness, while amine-capped CQD were deposited from a $100 \mathrm{mg} \mathrm{mL}^{-1}$ solution in octane, spun at $2500 \mathrm{rpm}$ to generate roughly $35 \mathrm{~nm}$ thick layers. Each layer was treated with $1 \% 3-$ mercaptopropionic acid (MPA) in methanol (v/v); for mutually-passivated films, the MPA solution included $0.2 \mathrm{mM} \mathrm{CdCl}_{2}$. All films prepared for XPS measurements (control and mutually-passivated) were exchanged with an $\mathrm{MPA} / \mathrm{CdCl}_{2}$ solution to maintain a consistent number of Cl-passivation steps. All films were rinsed with pure methanol after ligand exchange, spinning at $2500 \mathrm{rpm}$ after each rinse. For photovoltaic devices, top electrodes were deposited by thermal and electron beam evaporation, and consisted of $40 \mathrm{~nm}$ of $\mathrm{MoO}_{3}$, $50 \mathrm{~nm}$ of gold and $150 \mathrm{~nm}$ of silver, deposited at a rate of 0.2 (thermal $\mathrm{MoO}_{3}$ ), 0.4 (electron beam $\mathrm{Au}$ ) and 1 (thermal $\mathrm{Ag}) \AA \stackrel{\mathrm{A}}{\mathrm{s}}$, at a pressure of $<1 \times 10-6$ mbar.

Materials Characterization: The surface elements and chemical states of the PbS CQD films were analyzed using X-ray photoelectron spectroscopy (XPS) (PHI-5500). A monochromated Al K radiation source $(1486.7 \mathrm{eV})$ was used to excite photoelectrons in an ultrahigh vacuum atmosphere at ca. $10^{-9}$ Torr. The binding energy scale was calibrated using the Au $4 \mathrm{f} 7 / 2$ peak at $83.98 \mathrm{eV}$ and the $\mathrm{Cu} 2 \mathrm{p} \mathrm{3/2}$ peak of sputter-cleaned $\mathrm{Cu}$ at $932.67 \mathrm{eV}$.

Transient Photoluminescence: Transient PL measurements were performed in reflection geometry (at an incidence angle of $30^{\circ}$ from the sample surface, to avoid spurious reflections of the incident light) using a $633 \mathrm{~nm}$ laser diode as a pulsed excitation source (with a temporal profile of $\sim 0.13 \mathrm{~ns}$ ), in combination with a double-grating spectrometer. The time traces were acquired using a time-correlated single-photon-counting (TCSPC) near-infrared detector (aircooled photomultiplier tube). The time window was set according to the measured timescale, in order to ensure a complete decay of the photogenerated carriers between consecutive excitation pulses.

Photovoltaic Performance Characterization: Current-voltage data were measured with the device at ambient temperature in a constantly purged nitrogen environment, using a Keithley 
2400 source meter. The solar spectrum at AM1.5 was simulated to within class A

specifications (less than 25\% spectral mismatch) with a xenon lamp and filters (ScienceTech; measured intensity of $100 \mathrm{~mW} \mathrm{cm-2).} \mathrm{The} \mathrm{source} \mathrm{intensity} \mathrm{was} \mathrm{measured} \mathrm{with} \mathrm{a} \mathrm{Melles-Griot}$ broadband power meter through a circular $0.049 \mathrm{~cm}^{2}$ aperture. The same aperture was used in device measurements; the aperture is slightly smaller than the top device electrode to avoid overestimating photocurrent. The entire photon fluence passing through the aperture was counted as incident on the device for all current analyses. The spectral mismatch of the system was characterized using a calibrated reference solar cell (Newport). The total AM 1.5 spectral mismatch — taking into account the simulator spectrum and the spectral responsivities of the test cell, reference cell, and broadband power meter-was re-measured periodically and found to be ca. $9.4 \%$. This multiplicative factor, $M=0.906$, was applied to the current density values of the $\mathrm{J}-\mathrm{V}$ curve to most closely resemble true AM 1.5 performance $^{[30]}$. External quantum efficiency measurements were obtained by applying chopped $(220 \mathrm{~Hz})$

monochromatic illumination (450 W xenon lamp through a monochromator with ordersorting filters) collimated and cofocused with a 0.7 sun intensity white light source on the device of interest. The power was measured with calibrated Newport 818-UV and Newport 818-IR power meters. The response from the chopped signal was measured using a Stanford Research system current preamplifier feeding into a Stanford Research system lock-in amplifier set to voltage mode.

\section{Supporting Information}

Supporting Information is available from the Wiley Online Library or from the author.

\section{Acknowledgements}

This publication is based in part on work supported by Award KUS-11-009-21, made by King Abdullah University of Science and Technology (KAUST), by the Ontario Research Fund Research Excellence Program, and by the Natural Sciences and Engineering Research Council. GHC acknowledges funding support from the Vanier Canada Graduate Scholarship program. The authors thank Dr. F. Fan for TEM measurements, and R. Wolowiec and D. Kopilovic for technical assistance throughout these studies. 
Received: ((will be filled in by the editorial staff))

Revised: ((will be filled in by the editorial staff)) Published online: ((will be filled in by the editorial staff))

[1] G. Konstantatos, I. Howard, A. Fischer, S. Hoogland, J. Clifford, E. Klem, L. Levina, E. H. Sargent, Nature 2006, 442, 180.

[2] S. A. McDonald, G. Konstantatos, S. Zhang, P. W. Cyr, E. J. D. Klem, L. Levina, E. H. Sargent, Nat. Mater. 2005, 4, 138.

[3] A. H. Ip, S. M. Thon, S. Hoogland, O. Voznyy, D. Zhitomirsky, R. Debnath, L. Levina, L. R. Rollny, G. H. Carey, A. Fischer, K. W. Kemp, I. J. Kramer, Z. Ning, A. J. Labelle, K. W. Chou, A. Amassian, E. H. Sargent, Nat. Nanotechnol. 2012, 7, 577.

[4] C.-H. M. Chuang, P. R. Brown, V. Bulović, M. G. Bawendi, Nat. Mater. 2014, 13, 796.

[5] J. S. Steckel, S. Coe-Sullivan, V. Bulović, M. G. Bawendi, Adv. Mater. 2003, 15, 1862.

[6] L. Qian, Y. Zheng, J. Xue, P. H. Holloway, Nat. Photonics 2011, 5, 543.

[7] C. B. Murray, D. J. Norris, M. G. Bawendi, J. Am. Chem. Soc. 1993, 115, 8706.

[8] X. Wang, G. I. Koleilat, J. Tang, H. Liu, I. J. Kramer, R. Debnath, L. Brzozowski, D. A. R. Barkhouse, L. Levina, S. Hoogland, E. H. Sargent, Nat. Photonics 2011, 5, 480.

[9] O. E. Semonin, J. M. Luther, S. Choi, H.-Y. Chen, J. Gao, A. J. Nozik, M. C. Beard, Science 2011, 334, 1530.

[10] L. Cademartiri, E. Montanari, G. Calestani, A. Migliori, A. Guagliardi, G. A. Ozin, J. Am. Chem. Soc. 2006, 128, 10337.

[11] I. Moreels, K. Lambert, D. Smeets, D. De Muynck, T. Nollet, J. C. Martins, F. Vanhaecke, A. Vantomme, C. Delerue, G. Allan, Z. Hens, ACS Nano 2009, 3, 3023.

[12] J. Mooney, M. M. Krause, J. I. Saari, P. Kambhampati, Phys. Rev. B 2013, 87, 081201.

[13] P. Kambhampati, Chem. Phys. 2015, 446, 92.

[14] C. Giansante, L. Carbone, C. Giannini, D. Altamura, Z. Ameer, G. Maruccio, A. Loiudice, M. R. Belviso, P. D. Cozzoli, A. Rizzo, G. Gigli, J. Phys. Chem. C 2013, 117, 13305.

[15] D. Kim, D.-H. Kim, J.-H. Lee, J. Grossman, Phys. Rev. Lett. 2013, 110, 196802.

[16] J. M. Luther, M. Law, Q. Song, C. L. Perkins, M. C. Beard, A. J. Nozik, ACS Nano 2008, $2,271$.

[17] A. Fischer, L. Rollny, J. Pan, G. H. Carey, S. M. Thon, S. Hoogland, O. Voznyy, D. Zhitomirsky, J. Y. Kim, O. M. Bakr, E. H. Sargent, Adv. Mater. 2013, 25, 5742.

[18] Z. Ning, O. Voznyy, J. Pan, S. Hoogland, V. Adinolfi, J. Xu, M. Li, A. R. Kirmani, J.P. Sun, J. Minor, K. W. Kemp, H. Dong, L. Rollny, A. Labelle, G. Carey, B. Sutherland, I. Hill, A. Amassian, H. Liu, J. Tang, O. M. Bakr, E. H. Sargent, Nat. Mater. 2014, 13, 822.

[19] J. Tang, K. W. Kemp, S. Hoogland, K. S. Jeong, H. Liu, L. Levina, M. Furukawa, X. Wang, R. Debnath, D. Cha, K. W. Chou, A. Fischer, A. Amassian, J. B. Asbury, E. H. Sargent, Nat. Mater. 2011, 10, 765.

[20] D. Zhitomirsky, O. Voznyy, L. Levina, S. Hoogland, K. W. Kemp, A. H. Ip, S. M. Thon, E. H. Sargent, Nat. Commun. 2014, 5.

[21] W. J. Baumgardner, K. Whitham, T. Hanrath, Nano Lett. 2013, 13, 3225.

[22] C. S. S. Sandeep, J. M. Azpiroz, W. H. Evers, S. C. Boehme, I. Moreels, S. Kinge, L.

D. A. Siebbeles, I. Infante, A. J. Houtepen, ACS Nano 2014.

[23] N. C. Anderson, J. S. Owen, Chem. Mater. 2012, 25, 69.

[24] N. C. Anderson, M. P. Hendricks, J. J. Choi, J. S. Owen, J. Am. Chem. Soc. 2013, 135, 18536. 
[25] Z. M. Norman, N. C. Anderson, J. S. Owen, ACS Nano 2014, 8, 7513.

[26] P. E. Shaw, A. Ruseckas, I. D. W. Samuel, Adv. Mater. 2008, 20, 3516.

[27] S. D. Stranks, G. E. Eperon, G. Grancini, C. Menelaou, M. J. P. Alcocer, T. Leijtens, L. M. Herz, A. Petrozza, H. J. Snaith, Science 2013, 342, 341.

[28] G. Xing, N. Mathews, S. Sun, S. S. Lim, Y. M. Lam, M. Grätzel, S. Mhaisalkar, T. C. Sum, Science 2013, 342, 344.

[29] M. A. Hines, G. D. Scholes, Adv. Mater. 2003, 15, 1844.

[30] H. J. Snaith, Nat. Photonics 2012, 6, 337. 
Through a combination of chemical and mutual dot-to-dot surface passivation, high quality colloidal quantum dot solids have beenwere fabricated. The joint passivation techniques lead to a record diffusion length for colloidal quantum dots of $230145+/-2021$ $\mathrm{nm}$. The technique is applied to create thick photovoltaic devices that exhibit high current density without losing fill factor.

Graham H. Carey, Larissa Levina, Riccardo Comin, Oleksandr Voznyy, Edward H. Sargent*

Record Charge Carrier Diffusion Length in Colloidal Quantum Dot Solids via Mutual Dot-to-Dot Surface Passivation

ToC figure

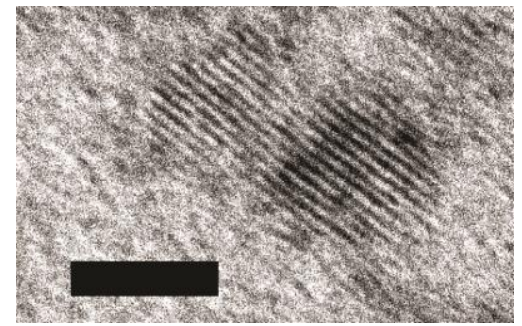

